\title{
On minimal blocking sets of the generalized quadrangle $Q(4, q)$
}

\author{
Miroslava Cimráková ${ }^{1}$ and Veerle Fack ${ }^{1}$ \\ ${ }^{1}$ Research Group on Combinatorial Algorithms and Algorithmic Graph Theory, Department of Applied Mathematics \\ and Computer Science, Ghent University, Krijgslaan 281-S9, B-9000 Ghent, Belgium. \\ E-mail: Miroslava.Cajkova@Ugent.be, Veerle.Fack@Ugent.be
}

The generalized quadrangle $Q(4, q)$ arising from the parabolic quadric in $P G(4, q)$ always has an ovoid. It is not known whether a minimal blocking set of size smaller than $q^{2}+q$ (which is not an ovoid) exists in $Q(4, q), q$ odd. We present results on smallest blocking sets in $Q(4, q), q$ odd, obtained by a computer search. For $q=5,7,9,11$ we found minimal blocking sets of size $q^{2}+q-2$ and we discuss their structure. By an exhaustive search we excluded the existence of a minimal blocking set of size $q^{2}+3$ in $Q(4,7)$.

Keywords: generalized quadrangle, blocking set, search algorithm

\section{Preliminaries}

A (finite) generalized quadrangle (GQ) is an incidence structure $S=(P, B, I)$ in which $P$ and $B$ are disjoint (nonempty) sets of objects called points and lines (respectively), and for which $I$ is a symmetric point-line incidence relation satisfying the following axioms:

(i) each point is incident with $1+t$ lines $(t \geq 1)$ and two distinct points are incident with at most one line;

(ii) each line is incident with $1+s$ points $(s \geq 1)$ and two distinct lines are incident with at most one point;

(iii) if $x$ is a point and $L$ is a line not incident with $x$, then there is a unique pair $(y, M) \in P \times B$ for which $x$ I $M I$ y $I L$.

We call the pair $(s, t)$ the order of this $\operatorname{GQ}(s, t)$. We denote collinear points $x$ and $y$ by $x \sim y$, and concurrent lines $L$ and $M$ by $L \sim M$. For $x \in P$ put $x^{\perp}=\{y \in P \| y \sim x\}$ and note that $x \in x^{\perp}$. If $A \subset P, A$ "perp" is defined by $A^{\perp}=\cap\left\{x^{\perp} \| x \in A\right\}$. For the theory of generalized quadrangles, we refer to [1].

An ovoid of $S$ is a set $\mathcal{O}$ of points of $S$ such that each line of $S$ is incident with a unique point of $\mathcal{O}$. An ovoid of a $\mathrm{GQ}(s, t)$ has size $s t+1$. A partial ovoid is a set $\mathcal{O}$ of points of $S$ such that each line of $S$ 1365-8050 @ 2005 Discrete Mathematics and Theoretical Computer Science (DMTCS), Nancy, France 
is incident with at most one point of $\mathcal{O}$. A partial ovoid is called maximal when it is not contained in a larger partial ovoid.

A blocking set of $S$ is a set $\mathcal{B}$ of points of $S$ such that each line of $S$ is incident with at least one point of $\mathcal{B}$. A blocking set $\mathcal{B}$ is minimal when no proper subset of it is still a blocking set. Necessarily $|\mathcal{B}| \geq s t+1$, while equality holds if $\mathcal{B}$ is an ovoid.

Let $\mathcal{B}$ be a blocking set of size $s t+1+r$. We call $r$ the excess of the blocking set. A line of $S$ is called a multiple line if it contains at least two points of $\mathcal{B}$. The excess of a line is the number of points of $\mathcal{B}$ it contains, minus one.

Two subspaces are skew if they have no point in common. A line is called a transversal of a set of pairwise skew subspaces if it intersects each subspace in exactly one point.

Let $P$ be a 3-dimensional projective space. A non-empty set $\mathcal{R}$ of pairwise skew lines of $P$ is called a regulus if:

1. Through each point of each line of $\mathcal{R}$ there is a transversal of $\mathcal{R}$.

2. Through each point of a transversal of $\mathcal{R}$ there is a line of $\mathcal{R}$.

If $P$ has finite order $q$ then any regulus consists of exactly $q+1$ lines. The set $\mathcal{R}^{\prime}$ of all transversals of a regulus $\mathcal{R}$ is itself a regulus, called the opposite regulus of $\mathcal{R}$. Let us call grid a regulus and its opposite regulus.

\section{Small minimal blocking sets of $Q(4, q)$}

Here we will consider the classical generalized quadrangle of order $(q, q)$ (with $q$ a power of a prime) arising from the non-singular 4-dimensional parabolic quadric $Q(4, q)$. It is known that $Q(4, q)$ always has an ovoid.

It is easy to see that the following construction gives a minimal blocking set of size $q^{2}+q$ : take a point $x$ of $Q(4, q)$ and all lines through this point, then all $q(q+1)$ points on these lines (except $x$ ) form a minimal blocking set of size $q^{2}+q$. Our aim is to find a minimal blocking set of size smaller than $q^{2}+q$, which is not an ovoid.

Considering minimal blocking sets of $Q(4, q), q$ even, the following result is known.

Theorem 2.1 (Eisfeld et al. [2]) Let $\mathcal{B}$ be a blocking set of the quadric $Q(4, q), q$ even. If $q \geq 32$ and $|\mathcal{B}| \leq q^{2}+1+\sqrt{q}$, then $\mathcal{B}$ contains an ovoid of $Q(4, q)$. If $q=4,8,16$ and $|\mathcal{B}| \leq q^{2}+1+\frac{q+4}{6}$, then $\mathcal{B}$ contains an ovoid of $Q(4, q)$.

No similar result is known for $q$ odd. It is even not known, whether or not $Q(4, q), q$ odd, has a minimal blocking set of cardinality $q^{2}+2$. In [3], the authors were able to solve this problem when $q$ is an odd prime.

Theorem 2.2 (De Beule, Metsch [3]) If $q$ is an odd prime, then $Q(4, q)$ does not have a minimal blocking set of size $q^{2}+2$.

In [4] the authors performed a computer search to exclude the existence of a minimal blocking set of $Q(4,7)$ of size $q^{2}+3$ satisfying a special property: 
Lemma 2.3 (De Beule, Hoogewijs, Storme [4]) There is no minimal blocking set $\mathcal{B}$ of size $q^{2}+3$ on $Q(4,7)$ such that there is one point of $Q(4,7)$ with $q+1$ lines on it being blocked by exactly three points of $\mathcal{B}$.

S. De Winter [7] constructed a minimal blocking set $\mathcal{B}$ of size $28=q^{2}+q-2$ of $Q(4,5)$. Now the following question occurs: Are there any other generalized quadrangles $Q(4, q)$ having a minimal blocking set of size $q^{2}+q-2$ ?

\section{Search algorithms}

A first class of algorithms are exhaustive algorithms, where we use a standard backtracking algorithm which tries to extend a "partial blocking set" (which is not a blocking set yet) by adding the points of a set $A$ of remaining allowed points in a systematic way. When reaching a point where the set $A$ is empty, a new minimal blocking set has been found.

In order to avoid going through every single "partial blocking set" of the generalized quadrangle, we use pruning strategies based on specific properties of the generalized quadrangle $Q(4, q)$ given in [2] and [5].

Another class of algorithms are heuristic algorithms, which turn out to be effective for exploring the spectrum of sizes for which minimal blocking sets exist.

A simple greedy algorithm builds a minimal blocking set step by step, by removing points from a set of all points, until this set is a minimal blocking set. Several strategies are possible for choosing a point to be removed in each step. For instance, removing a point that leaves the largest number of points in the allowed set, will tend to build small minimal blocking sets. Starting from a minimal blocking set obtained by one of the above approaches, a simple restart strategy adds some of the points and again removes points until the blocking set is minimal. Both the adding and the removing can be done either randomly or following one of the above heuristics.

\section{Results}

Using the exhaustive algorithm described in Section 3, we obtain the following result:

Lemma 4.1 There is no minimal blocking set $\mathcal{B}$ of size $q^{2}+3$ on $Q(4,7)$.

Using the heuristic strategies from Section 3 we obtain minimal blocking sets of size $q^{2}+q-2$ (i.e. with excess $r=q-3$ ) for $q=5,7,9,11$. Hence, for small values of $q$, minimal blocking sets (which are not ovoids) of size smaller than $q^{2}+q$ do exist.

We now investigate the structure of these blocking sets. This is also done by a computer search. We focus on the multiple lines of the blocking sets and the way, in which they are structured. The case $q=9$ is different and we will mention it separately.

For $q=5,7,11$ we observed that, for the blocking sets found, all multiple lines have excess one, which means that the multiple lines contain two points of the blocking set.

Already S. De Winter noticed that for $q=5$ all multiple lines form a grid. We observed that also for $q=7,11$ grids are formed by the multiple lines; moreover, more than one grid appears (see Table 1). For 


\begin{tabular}{|l|c|c|c|}
\hline & \# all multiple lines & $r$ & \# grids \\
\hline \hline$Q(4,5)$ & 12 & 2 & 1 \\
$Q(4,7)$ & 32 & 4 & 2 \\
$Q(4,11)$ & 96 & 8 & 4 \\
\hline
\end{tabular}

Tab. 1: Structure of blocking sets

these cases the number of multiple lines is $2(q+1)(q-3) / 2$ and the number of grids formed by multiple lines is $(q-3) / 2$.

Further, for $q=7,11$ there are $q+1$ points of $\mathcal{B}$ common to all grids.

Finally we observed that it is possible to obtain a maximal partial ovoid of size $q^{2}-1$ by removing these $q+1$ common points and adding two points of the "perp" of this set. In $Q(4,5)$ are there 4 possibilities of obtaining such a maximal partial ovoid by removing $q+1$ points.

For $q=9$ we observed that, for the maximal blocking sets found, all multiple lines have excess three. The blocking set found has 20 multiple lines and we checked that they form a grid.

As mentioned in [6] we found by exhaustive search that no maximal partial ovoids of size $q^{2}-1$ exist in $Q(4,9)$.

To finish this paper we want to mention our current effort. We try to find a blocking set of size $q^{2}+q-2$ for larger $q$, especially for $q=13$. If we suppose that this blocking set would be of the same structure, it should contain 5 grids with 14 points in the intersection. So far we have found no blocking set of the given size by our (not exhaustive!) computer search. We expect that no blocking set of $Q(4,13)$ of size $q^{2}+q-2$ exists.

\section{References}

[1] S.E. Payne and J.A. Thas. Finite Generalized Quadrangles. Pitman Res. Notes Math. Ser. 110. Longman, 1984.

[2] J. Eisfeld, L. Storme, T. Szönyi, and P. Sziklai. Covers and blocking sets of classical generalized quadrangles. Discrete Math.,238:35-51, 2001.

[3] J. De Beule, K. Metsch. Minimal blocking sets of size $q^{2}+2$ of $Q(4, q), q$ an odd prime, do not exist. Finite Fields Appl., to appear.

[4] J. De Beule, A. Hoogewijs, L. Storme. On the size of minimal blocking sets of $Q(4, q)$, for $q=5,7$ ACM SIGSAM Bulletin, 38:67-84, 2004.

[5] J. De Beule. Blocking sets and partial spreads in finite polar spaces. $\mathrm{PhD}$ thesis, Gent University, 2004.

[6] M. Cimráková, S. De Winter, V. Fack, L. Storme. On the smallest maximal partial ovoids and spreads of the generalized quadrangles $W(q)$ and $Q(4, q)$. J. Comb. Theory A, submitted, 2005.

[7] S. De Winter. Private communication. 